\title{
ORIGINAL RESEARCH

WALKING CAPACITY AND FALLS-EFFICACY CORRELATES
WITH PARTICIPATION RESTRICTION IN INDIVIDUALS
WITH CHRONIC STROKE: A CROSS SECTIONAL STUDY

\author{
${ }^{1}$ Ms. Neelam Nayak \\ ${ }^{2}$ Pranali Mahajan
}

\section{ABSTRACT}

Background: Mobility impairments seen after Stroke impact walking speed, endurance and balance. Almost all the individuals with Stroke have fear of fall. The physical impairments in balance and gait along with individual's perception about his/her own abilities to maintain balance might have an impact on level of activity and participation in the community. The association of these variables with recovery of Stroke has been well studied. However, it is currently unknown which of these variables are most associated with activity and participation in the community. This study aimed to identify the correlation of walking capacity and perception of fall with activity $\mathcal{E}$ participation.

Methods: 30 Subjects were assessed for - walking capacity (6 minute walk test) \& Self-efficacy for falls (Modified Falls Efficacy scale). Level of Activity Limitation (AL) \& Participation Restriction (PR) was graded on validated ICF Measure of Participation and Activities. (IMPACT-S)

Results: Data was analyzed using Pearson's correlation coefficient $\&$ regression model. Walking distance and Falls-efficacy is significantly correlated $(r=-0.751$ and -0.683 , respectively) with Participation restriction. Walking distance correlated with Activity Limitation $(r=-0.714)$ significantly. Falls efficacy has a correlation coefficient of -0.642 with Activity Limitation. When put into Regression models, Walking Capacity \& Gait Velocity was found to be independently associated with AL \&PR.

Conclusion: There is significant relationship between falls self-efficacy, walking capacity and Post-stroke activity $\&$ participation. Participation can be impacted by factors such as self-motivation and confidence about one's balance abilities. This is reflected by the correlation between falls efficacy and participation. Physical parameters such as the distance walked can contribute to participating in the community, and can predict variation in AL-PR

Keywords: Falls efficacy, Activity Limitation \& Participation restriction, IMPACT(S)

Received 21 ${ }^{\text {st }}$ August 2014, revised $23^{\text {rd }}$ September 2014, accepted $07^{\text {th }}$ October 2014

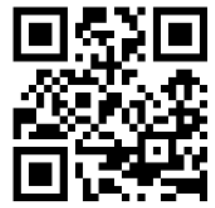

WWW.ijphy.org
DOI: $10.15621 / \mathrm{ijphy/2015/v2i1/60032}$

\footnotetext{
${ }^{2}$ Lecturer,

MGM College of Physiotherapy,

Navi Mumbai, India.
}

\section{CORRESPONDING AUTHOR}

\section{${ }^{1}$ Ms. Neelam Nayak}

Lecturer,

MGM College of Physiotherapy,

Navi Mumbai, India. 


\section{INTRODUCTION}

Stroke is currently the leading cause of neurological disability in adults. The Global Burden of Disease Study estimated a population-based annual stroke incidence in India to be $89 / 100,000$ in 2005, which is projected to increase to 91/100,000 in 2015(WHO-2004). ${ }^{1}$

Irrespective of the type and course of recovery of Stroke, there are certain mobility impairments such as affection in Balance, altered gait pattern, variations in Gait speed that restrict the individuals with Stroke in their level of Participation in the community and Activities of Daily Living.

The International Classification of Functioning, Disability and Health (ICF) defines participation as "the involvement in a life situation" and participation restrictions as "problems an individual may experience in involvement in life situations". Activity is the performance of a task or action. ${ }^{2}$ Many studies have described determinants of Participation after stroke and have established a significant association between Activity Limitation \& Participation Restriction with the risk of recurrent Stroke. ${ }^{(3-9)}$ Mayo, et al concluded that 50\% of the community-dwelling stroke population is living with sequelae of stroke that place them at risk for a diminished activity level and social isolation that can result in further negative health events. ${ }^{10}$

One of the primary impairments post-stroke is reduced walking capacity. Poor performance in long-distance walking is associated with mortality and incident cardiovascular disease in older adults. Fall-related self-efficacy (Subjective Measure) is a psychological characteristic, based on the selfefficacy concept, which can have an influence on functional decline, as fear may limit a person's function to an extent beyond that due to an underlying impairment of physical ability alone. ${ }^{11}$ Impaired postural control, reactive and anticipatory balance can produce a significant fear of fall post-Stroke. These impairments have a potential to limit one's ability to be an active participant in society as well as at home. It is essential for Physiotherapists to know the impact of these factors on function and participation, to achieve the desired functional goals for individuals post Stroke and to enhance the recovery in view of returning to their pre-morbid functional role.

Certain studies have focused on comparing the Subjective and Objective measures that can be related to Activity limitation and Participation Restriction. Robinson and Shumway-Cook have recommended in their study the need to examine the role of personal factors, especially balance selfefficacy, in recovery of walking related participation following stroke. ${ }^{12}$ We felt a need to put light on this area as the variability in Indian environment may have a definite impact on walking (mechanical barriers, uneven terrains etc.) and consequently on the fear of fall. Details of structural differences in these areas are beyond the scope of this study. We decided to explore the association between Walking Capacity, Gait Velocity \& Falls efficacy with Activity limitation and Participation Restriction (ALEPR), as there is a paucity of research in an Indian context.

We focused on following questions to investigate this area of research -

1. Does walking capacity and fear of fall (fallefficacy) affect Activities and Participation in individuals with Stroke?

2. Does walking distance and velocity (Objective measure) and falls-efficacy (Subjective measure) predict the level of Activities and Participation?

3. What are the other factors that can determine the level of Activity limitation and Participation Restriction?

\section{METHODOLOGY}

\section{Design}

This was a prospective cross sectional study, conducted in Neuro-rehabilitation Out-patient Department; over a period of 5 months. Prior approval of College Ethic committee was obtained.

\section{Participants, therapists, centers}

30 individuals coming to OPD were recruited by a convenient sampling method. They were explained about the purpose of the study and Informed consent was obtained prior to assessment

Subjects were recruited in the study based on the following criteria.

1. Clinical diagnosis of Stroke

2. Ability to comprehend commands (Screened on MMSE)

3. Duration of onset $>$ More than 6 months (i.e. Chronic Stroke)

4. Minimum Score of 22/56 on Berg Balance Scale

5. Ability to walk with/without support.

Data was collected by a single trained therapist.

Procedure: Impairments were assessed as follows:

Walking capacity was measured with the 6-minute walk, a valid and reliable measure. ${ }^{13-16}$ 
Each participant was instructed to walk at a comfortable pace on a $10 \mathrm{~m}$ measured walkway for 6 minutes. Walking capacity was measured as the total distance walked during the 6 minutes (meters/second)

Gait Velocity was assessed on 10 meter walk test. Subjects were allowed to walk with assistive devices, if any. They walked at self-selected speed.

Falls efficacy was measured using the Modified Falls Efficacy Scale (MFES); this is a 14-item selfreport scale measuring confidence in one's ability to avoid falling during the performance of activities of daily living (ADL). ${ }^{17}$

Subjects were asked to rate their confidence in performing each activity without falling on a $0-10$ scale, the average score across all 14 items was taken, with a minimum score of 0 indicating no confidence and a maximum score of 10 indicating full confidence (high falls efficacy) in performing the tasks without falling.

Activity and participation was assessed on the validated ICF Measure of Participation and Activities (IMPACT). ${ }^{18}$ The IMPACT is a 33-item scale designed to identify restrictions a person has in the 9 categories of activity and participation outlined by the ICF. IMPACT-S scale (ICF Measure of Participation and Activities Questionnaire) was used for evaluating activity limitation and participation restriction based on the following nine domains i.e. (1) Learning and applying knowledge; (2) General tasks and demands; (3) Communication; (4) Mobility and; (5) Self-care. Participation chapters are: (6) Domestic life; (7) Interpersonal interactions and relationships; (8) Major life areas; (9) Community, social and civic life.

The 9 category scores can be totaled to obtain a global measure of activity and participation or computed independently as a measure of specific activity (items 1-19) or participation (items 20-33). Scoring ranges from 19 to 76 for activity, 14 to 56 for participation, or 33 to 132 for the total combined score. Decreased scoring indicates fewer limitations (higher function) in activity and participation. ${ }^{19}$

\section{RESULTS}

Our research question was answered by calculating correlations and applying logistic regression. First, the descriptive data was collated. Next, Pearson correlation coefficients for Walking Capacity, Gait velocity and MFES and ICF components (ALEPR) were calculated. For each association, a correlation coefficient $(r)$ and a $P$ value were calculated.
Regression analysis to study the variance was calculated for significantly correlated variables. Data were graphed and examined to verify normality of distribution.

Number of participants was determined prior to the commencement of study by setting CI of $95 \%$, $\mathrm{Z}=1.96$.

Mean age of the Participants was 55.76 years (SD1.82)

In the sample space of 30 there were $24(80 \%)$ males and 6(20\%) females Mean duration PostStroke was 10.43 months (SD 1.86)

18(60\%) were Right hand dominant and 12 (40\%) were left hand dominant.

All the subjects recruited were on Physiotherapy treatment. Mean duration of the treatment program was 4 months (SD 0.59). 14 subjects $(46.66 \%)$ reported one fall in last 6 months or since the episode of stroke. Only one patient was using an Ankle Foot Orthosis (AFO).

Walking parameters:

Mean distance walked is 125.3 Meters (SD1.21)

Mean Gait velocity is $0.46 \mathrm{~m} / \mathrm{s}$. (SD0.30)

Falls -efficacy:

Mean score for MFES is 102.72 (SD 24)

Activity \& Participation:

ICF Activity limitation and Participation Restriction has 9 chapters. First 5 chapters were included for Activity limitation and next 4 chapters as Participation Restriction. Mean for activity limitation is 33 and mean for Participation Restriction is 27.83

Research question 1:

Does walking capacity and fear of fall (fall-efficacy) affect Activities and Participation in individuals with Stroke?

Walking Capacity \& Activity Limitation:

6 MWT distance is correlated with Activity limitation ( $r=-0.714$, at 95\% CI 0.70-0.80).

Gait velocity is correlated with Activity limitation ( $\mathrm{r}=-0.677$, at 95\% CI 0.60-0.71) Walking Capacity $\mathcal{E}$ Participation Restriction:

6 MWT distance is correlated with Participation Restriction ( $\mathrm{r}=-751$, at 95\% CI 0.85-0.96) Gait velocity is correlated with Participation Restriction ( $\mathrm{r}=-0.671$, at 95\% CI 0.65-0.88) Demographics and ALEPR were not significantly correlated. 
Falls-efficacy \& Activity limitation: MFES is correlated to both AL $(r=-0.642) \&$ PR $(=-0.683)$

Research question 2:

Can any of these impairments predict the level of Activities and Participation in individuals with Stroke? Walking capacity, gait speed and MFES were included in regression models. Refer Table 2.

Research question 3:

What are the other factors that can determine the level of Activity limitation and Participation Restriction?

The other factors like duration post-stroke, dominance were not correlated significantly.

\section{CONCLUSION \& DISCUSSION:}

This study highlights the importance of role of subjective and objective measure in level of participation. Subjectively, individuals' confidence in his abilities contributes to his motivational levels for being an active participant in the society. Out of the subjects (14) having history of falls, 8 (57.24\%) reported "no confidence at all" in simple shopping. For subjects who had no history of fall, 81.25\% (13) reported poor confidence in "using public transport". These findings strengthen the association between low confidence and participation in society. A systematic review by Korpershoek C concluded that Patients with high self-efficacy are functioning better in daily activities than patients with low self-efficacy. Cardiovascular fitness is very low in population with stroke. In our study, the mean gait velocity was found to be $0.46 \mathrm{~m} / \mathrm{s}$. Patterson et al found that walking capacity is largely determined by cardiorespiratory fitness $\left(R^{2}=0.26\right)$ for those who can walk faster (gait velocity $>0.48 \mathrm{~m} / \mathrm{sec}$ ) whereas walking capacity is more influenced by balance ability for slow walkers (gait velocity $<0.48$ $\mathrm{m} / \mathrm{sec}$ ). One of the limitations of our study is that we didn't take into consideration Objective evaluation of Balance, so we cannot say what exactly is the

contribution of Balance in reducing the walking capacity. ${ }^{20}$ Perry at el classified gait speed $<0.4 \mathrm{~m} / \mathrm{sec}$ as "household ambulation". Subjects walking $<0.4 \mathrm{~m} / \mathrm{s}$ are at a risk of fall ${ }^{21}$. This associated with fear of fall contributes to restriction of walking and other activities as well.

Walking capacity was independently associated with both AL and PR (refer Table 2). The link between reduced walking capacity and participation can be two-ways. It has been shown that patients with lower level of participation do have more fatigue, further affecting the cardiovascular endurance levels. ${ }^{22}$ Michael KM et al concluded in their study that reduced VO2 peak and metabolic fitness reserve are strongly correlated to balance $(\mathrm{r}=.374, \mathrm{P}=.02 ; \mathrm{r}=-.430, \mathrm{P}<$ .01 , respectively. $)^{23}$

In addition to physical parameters, psychological measure such as balance confidence may also influence walking capacity among individuals with chronic stroke. ${ }^{24}$ In our study MFES was weak predictor of variance for ALEPR. Though there is a significant correlation between MFES and AL-PR, when put into regression analysis, the predictability of MFES for ALEPR could not be established. It is possible that subjects may have over/underestimated their confidence in items such "using public transport", "crossing road", as there is a huge amount of diversity in these areas, depending from which socio-economic strata of India the subject comes from. When reporting Subjective evaluation, previous history of fall, self esteem and other personal factors can vary the subjects' interpretation about his abilities. This is supported by Schmidt's finding that anxiety, more than balance or a prior fall, was related to decreased falls self-efficacy, again identifying the importance of the psychological factors on post stroke recovery. ${ }^{25}$ It is even possible that Chronic Stroke subjects do not "set" their target as high for level of functioning and may come to terms with current level of activity and participation (mean duration Post-stroke was 10.43 months). Participation and level of activities may not be predicted based solely on self efficacy measures.

There is a limited generalisability of our study, as the sample size is limited. All the subjects were having score of more than 22/56 on Berg's Balance Scale, meaning they were at a "medium risk of fall". This might have had an effect on the scores of MFES. Subjects were on physiotherapy treatment and the level of ALEPR will definitely differ depending on the duration since Stroke and the recovery rate. Further research is needed to investigate the level of ALEPR based on the rate of recovery; the duration of treatment along with evaluation of subjective and objective parameters.

Gait training programs are well-emphasized in treatment protocols, therapist need to take into consideration the effect of these programs on level of AL-PR. At the same time, therapists need to focus on improving self efficacy to enhance the confidence for participating in the community.

\section{REFERENCES}

1. The global burden of disease: 2004 update. Geneva, World Health Organization, 2008 
2. International Classification of Functioning, Disability and Health: ICF. Geneva, Switzerland: World Health Organization;2001.

3. SchepersVP, Visser-Meily AM, KetelaarK, LindemanE. Prediction of social activity 1 year poststroke.Arch Phys Med Rehabil. 2005;86(7):1472-1476.

4. Hartman-Maeir A, Soroker N, Ring H, Avni $\mathrm{N}$, Katz N. Activities, participation and satisfactionone-year post stroke. Disabil Rehabil. 2007,29(7):559-566.

5. Desrosiers J, Demers L, Robichaud L, Vincent C,Belleville S, Ska B. Short-term changes in andpredictors of participation of older adults afterstroke following acute care or rehabilitation. Neurorehabil Neural Repair. 2008;22(3):288-297.

6. Chau JPC, Thompson DR, Twinn S, Chang AM,Woo $j$. Determinants of participation restrictionamong community dwelling stroke survivors: apath analysis. BMC NeuroL 2009;9:49.

7. Baseman S, Fisher K, Ward L, BhattacharyaA. The relationship of physical function tosocial integration after stroke. Neurosci Nurs.2010,42(5):237-244.

8. Dalemans RJP, De Witte LP, Beurskens AJHM, Vanden Heuvel WJA, Wade DT. An investigation intothe social participation of stroke survivors withaphasia. Disabil Rehabil. 2010;32(20):1 678-1685.

9. Danielsson A, Willen C, Sunnerhagen KS. Iswalking endurance associated with activity andparticipation late after stroke? Disabil Rehabil.2011 ;33(21-22):205 3-2057.

10. Nancy E. Mayo, Sharon Wood-Dauphinee, Robert Côté, Liam Durcan, Joseph Carlton, Activity, participation, and quality of life 6 months Arch Phys Med Rehabil 2002:83,(8) 1035-1042

11. Tinetti ME, Mendes de Leon CF, Doucete JT, Baker DJ. Fear of falling and fall related efficacy in relationship to functioning among community-living elders. J Gerontol 1994; 49: M140-147

12. Robinson CA, Shumway-Cook A,Ciol MA, Kartin D. Participation in community walking following stroke: subjective versus objective measures and the impact of personal factors. Phys Ther.2011; 91:1865-1876.]

13. Pohl P, Duncan P, Perera S, et al. Influence of stroke-related impairments in performance in 6-minute walk test. J Rehabil Res Dev 2002;39:439-44
14. Guyatt GH, Sullivan MJ, Thompson PJ, et al. The 6-minute walk: a new measure of exercise capacity in patients with chronic heart failure. Can Med Assoc J 1985; 132:919-23.

15. Enright P, Sherrill D. Reference equations for the six-minute walk in healthy adults. Am J Respir Crit Care Med 1998; 158:1384-7.

16. Enright PL, McBurnie MA, Bittner V, et al. The 6-min walk test: a quick measure of functional status in elderly adults. Chest 2003; 123:387-98.

17. Hill K,Schwarz J,et al.Fear of falling revisited, Arch Phys Med Rehabil 1996;77:1025-1029

18. Post MW, de Witte LP, Reichrath E, Verdonschot MM, Wijlhuizen GJ, Perenboom RJ. Development and validation ofIMPACT-S, an ICF-based questionnaire to measure activities and participation. J Rehabil Med 2008;40:620-7.

19. Stucki G, Cieza A, Ewert T, Kostanjsek N, Chatterji S, Üstün TB. Application of the International Classification of Functioning, Disability and Health (ICF) in clinical practice. Disabil Rehabil 2002; 24: 281-282.

20. Patterson SL, Forrester LW, Rodgers MM, Ryan AS, Ivey FM, Sorkin JD,et al. Determinants of walking function after stroke: differences by deficit severity. Arch Phys Med Rehabil 2007; 88: 115-119.

21. Tilson JK et al.Characterizing and Identifying Risk for Falls in the LEAPS Study: A Randomized Clinical Trial of Interventions to Improve Walking Post stroke. Stroke, 2012 Feb; 43 (2): 446-52.

22. Yang SY, Kong KH Level and predictors of participation in patients with stroke undergoing inpatient rehabilitation. Singapore Med J2013; 54 (10):564-8

23. Michael KM; Allen JK; Macko RF Reduced ambulatory activity after stroke: the role of balance, gait, and cardiovascular fitness. Arch Phys Med Rehabil 2005; 86(8): 1552-6.

24. Salbach NM, Mayo NE, Robichaud-Ekstrand S, Hanley JA,Richards CL, Wood-Dauphinee S. Balance self-efficacy and itsrelevance to physical function and perceived health status afterstroke. Arch Phys Med Rehabil 2006; 87: 364-370

25. Schmid AA, Van Puymbroeck M, Knies K, et al. Fear of falling among people who have sustained a stroke: a 6 -month longitudinal pilot study. Am J Occup Ther 2011; 65:125-32.

26. Korpershoek C, van der Bijl J, Hafsteinsdóttir TB. Self-efficacy and its influence on recovery of patients with stroke: a systematic review. J Adv Nurs. 2011;67(9):1876-94 
Table 1: Correlation statistics for dependent and independent variables

\begin{tabular}{|c|c|c|c|c|}
\hline Variable & Activity & Limitation & Participation & Combined ALEPR \\
\hline & (Total) $r$ & & Restriction (Total) r & \\
\hline MFES & -0.642 & & -0.683 & -0.679 \\
\hline 6MW -Distance & -0.714 & & -0.751 & -0.749 \\
\hline Gait Velocity & -0.677 & & -0.671 & -0.690 \\
\hline
\end{tabular}

Table 2: Regression analysis for dependent and independent variables

\begin{tabular}{|c|c|c|c|c|c|}
\hline Variable & Activity & Participatior & & Combined & CI 95\% \\
\hline & Limitation B & Restriction & B & ALEPR B & \\
\hline MFES & -0.203 & -0.251 & & -0.289 & $0.312-0.086$ \\
\hline 6MW -Distance & -0.410 & -0.459 & & -0.451 & $0.247-0.021$ \\
\hline Gait Velocity & -0.469 & -0.413 & & -0.487 & $0.243-0.093$ \\
\hline
\end{tabular}

\section{Citation}

Neelam Nayak; Pranali Mahajan. (2015). WALKING CAPACITY AND FALLS-EFFICACY CORRELATES WITH PARTICIPATION RESTRICTION IN INDIVIDUALS WITH CHRONIC STROKE: A CROSS SECTIONAL STUDY. International Journal of Physiotherapy, 2(1), 311-316. 